\title{
Stability of a Horizontal Fluid Interface in a Periodic Vertical Electric Field
}

\author{
ChIA-Shun YiH \\ Department of Engineering Mechanics, The University of Michigan, Ann Arbor, Michigan
}

(Received 4 January 1968)

\begin{abstract}
The stability of the interface in the presence of a periodic electric field is considered. It is shown that the stability is governed by a Mathieu equation, that the interface can be unstable even if the electric field is at all times weaker than that needed for instability in the case of a steady field, and that, when instability occurs, the waves may either be synchronous with the electric field, or have twice its frequency.
\end{abstract}

\section{INTRODUCTION}

The stability of a horizontal fluid interface between a conducting and a nonconducting fluid in a steady vertical electric field was treated by Taylor and McEwan. ${ }^{1}$ In their paper the forces arising from the electric field, from gravity, and from surface tension are balanced at the interface, and a stability criterion is found from this balance. Although the hydrodynamics of the conducting fluid was not considered in the paper by Taylor and McEwan, identical results are obtained if it is taken into consideration.

In this paper the instability of the interface in a vertical electric field varying periodically with time is considered. Because of the time dependence of the electric field, the simple equation of force balance can no longer be utilized to obtain the stability criterion, and the hydrodynamics of the fluid or fluids must be taken into account. When this is done, and the viscous effects are negligible, the stability of the interface can be shown to be governed by a Mathieu equation whose coefficients depends on the gravitational acceleration, the surface tension, the magnitude and frequency of the periodic electric field, the depth (or depths), and the geometry of the container.

\section{THE EQUILIBRIUM STATE}

Suppose that the upper fluid which can be a gas or a liquid, is nonconductive and the lower fluid, which is invariably a liquid, is conductive of electricity. The depth of the upper fluid is denoted by $h_{1}$ and that of the lower fluid by $h_{2}$. (See Fig. 1) The upper fluid, which is bounded above by an electrode with potential $V_{1}$ and below by the interface, has depth $h_{1}$, and the lower fluid, bounded below by an electrode with potential

$$
V_{2}=V_{0} \cos \omega t
$$

${ }^{1}$ G. I. Taylor and A. D. McEwan, J. Fluid Mech. 22, 1 (1965).

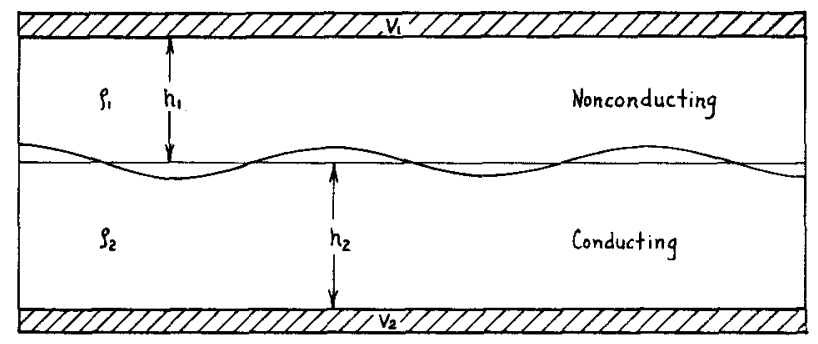

FIg. 1. Definition sketch.

has depth $h_{2}$. In Eq. (1) $t$ is the time and $\omega$ the circular frequency of $V_{2}$. (Note that the usage of $V_{0}$ in this paper is different from that in the paper by Taylor and McEwan.) We will assume that the Reynolds number based on either the depth or the wavelength of the disturbance to be large for either fluid, so that viscous effects are negligible.

If the densities of the upper and lower fluids are denoted by $\rho_{1}$ and $\rho_{2}$, respectively, and the gravitational acceleration by $g$, the hydrostatic pressure distributions in the two fluids are given by

$$
\begin{aligned}
& p_{1}=p_{0}-g \rho_{1} z, \\
& p_{2}=p_{0}-\frac{K}{8 \pi}\left(\frac{V_{1}-V_{2}}{h_{1}}\right)^{2}-g \rho_{2} z,
\end{aligned}
$$

if $z$ is measured vertically from the interface, and $K$ is the dielectric constant of the nonconducting fluid. The pressure in the upper fluid at the interface is denoted by $p_{0}$. It can be assigned any arbitrary value without affecting anything, since both fluids can be considered incompressible. Note that the pressure $p_{2}$ at the interface (or just below it) is not $p_{0}$ but $p_{0}$ reduced by the electric part of the Maxwell stress normal to the interface.

The electric potential $V$ is simply $V_{2}$ in the conducting fluid, so that the electric field in that fluid is zero. In the nonconducting fluid

$$
V=V_{2}+\frac{V_{1}-V_{2}}{h_{1}} z,
$$


so that the field in that fluid is

$$
E_{1}=\frac{V_{1}-V_{2}}{h_{1}}
$$

We assume that the horizontal dimensions of the container are much larger than $h_{1}+h_{2}$, so that surface tension effects are negligible over the main part of the interface.

\section{FORMULATION OF THE STABILITY PROBLEM}

Suppose that the interface of the two fluids is slightly displaced, so that at any time it is described by the equation

$$
z=\zeta=a(t) f(x, y)
$$

in which $f$ satisfies the equation

$$
\left(\frac{\partial^{2}}{\partial x^{2}}+\frac{\partial^{2}}{\partial y^{2}}+k^{2}\right) f=0
$$

$k^{2}$ being an eigenvalue which makes $\partial f / \partial n=0$ ( $n$ is the normal distance) at the wall of the cylindrical container and is thus dependent on geometry alone. The electric field and potential in the lower fluid are still given by

$$
E_{2}=0 \text { and } V_{2}=V_{0} \cos \omega t .
$$

But the electric potential of the upper fluid, which must satisfy the Laplace equation and the boundary conditions (if we now specify $V_{1}=-V_{2}$ )

and

$$
V=V_{1}=-V_{2} \text { at } z=h_{1}
$$

is given by

$$
V=V_{2} \text { at } z=\zeta
$$

$$
V=V_{2}\left[1-\frac{2 z}{h_{1}}-\frac{2 \sinh k\left(z-h_{1}\right)}{h_{1} \sinh k h_{1}} a(t) f(x, y)\right] \text {. }
$$

The normal component of the electric stress (tensile) at the interface is, with $K$ indicating the dielectric constant,

$$
\frac{K}{8 \pi}\left\{\left(\frac{\partial V}{\partial x}\right)^{2}+\left(\frac{\partial V}{\partial y}\right)^{2}+\left(\frac{\partial V}{\partial z}\right)^{2}\right\},
$$

since $z=f$ is a surface of constant potential $V_{2}$ (although it varies with time). This stress component is

$$
\sigma_{e n}=\frac{K}{2 \pi} \frac{V_{2}^{2}}{h_{1}^{2}}\left[1+2 k \operatorname{coth}\left(k h_{1}\right) a(t) f\right],
$$

if terms quadratic in $a(t)$ are neglected. The subscript $e$ indicates that $\sigma_{\sigma}$ is an electric stress, and the subscript $n$ indicates the normal component.

Now turning to the hydrodynamics of the fluids, we note that since the lower fluid has no electric field or magnetic field, its motion is governed by the ordinary hydrodynamic equations. If we assume that the Reynolds number (based on any representative length) is large, the fluid may be treated as inviscid, and if the motion is assumed to have begun from rest it is irrotational. Hence the potential $\phi_{2}$ for the motion of the lower fluid is

$$
\phi_{2}=A_{2}(t) \cosh k\left(z+h_{2}\right) f(x, y)+G_{2}(t),
$$

which satisfies the boundary conditions

$$
\frac{\partial \phi_{2}}{\partial n}=0 \quad \text { at the wall, } \quad \frac{\partial \phi_{2}}{\partial z}=0 \quad \text { at } z=-h_{2} .
$$

Similarly, the upper fluid, which is free of electric charges, has a constant $K$, and therefore possesses a velocity potential

$$
\phi_{1}=A_{1}(t) \cosh k\left(z-h_{1}\right) f(x, y)+G_{1}(t),
$$

which satisfied

$$
\frac{\partial \phi_{1}}{\partial n}=0 \quad \text { at the wall and } \quad \frac{\partial \phi_{1}}{\partial z}=0 \quad \text { at } \quad z=h_{1} .
$$

We are left to deal with the interfacial conditions. There are two such conditions, one kinematic, and the other dynamic. The kinematic condition is

$$
\frac{\partial \phi_{1}}{\partial z}=\frac{\partial \phi_{2}}{\partial z}=\frac{d a(t)}{d t} f(x, y) \text { at } z=0,
$$

and the dynamic condition is

$$
\begin{aligned}
p_{2}-p_{1} & +\frac{K}{8 \pi}|E|^{2} \\
& =-T a(t)\left(\frac{\partial^{2}}{\partial x^{2}}+\frac{\partial^{2}}{\partial y^{2}}\right) f=k^{2} T a(t) f,
\end{aligned}
$$

$T$ being the surface tension and $p$ the pressure.

Equations (13) give

$-A_{1}(t) k \sinh k h_{1}=\frac{d a(t)}{d t}=A_{2}(t) k \sinh k h_{2}$.

In order to utilize (14), it is necessary to use the Bernoulli equations

$$
\begin{aligned}
& \rho_{1}\left(\frac{\partial \phi_{1}}{\partial t}+\frac{1}{2} q_{1}^{2}\right)+p_{1}-\rho_{1} g z=F_{1}(t), \\
& \rho_{2}\left(\frac{\partial \phi_{2}}{\partial t}+\frac{1}{2} q_{2}^{2}\right)+p_{2}-\rho_{2} g z=F_{2}(t),
\end{aligned}
$$

in which $q$ is the speed. Since an arbitrary function of time has been added to $\phi_{1}$ and to $\phi_{2}$, we can take $F_{1}(t)$ and $F_{2}(t)$ to be zero. Neglecting $q^{2}$, we have

$$
p_{2}-p_{1}=g z\left(\rho_{2}-\rho_{1}\right)+\rho_{1} \frac{\partial \phi_{1}}{\partial t}-\rho_{2} \frac{\partial \phi_{2}}{\partial t} .
$$

Putting Eqs. (18), (11), and (12) into Eq. (14), and 
setting $z$ equal to $\zeta$ in (18) and evaluating $\phi_{1}$ and $\phi_{2}$ in Eq. (18) by Eqs. (11) and (12), putting the result into Eq. (14), and using the $\sigma_{e n}$ in (10) for the electric term in (14), we have, besides

$$
\frac{K}{2 \pi} \frac{V_{2}^{2}}{h_{1}^{2}}=\rho_{2} G_{2}^{\prime}(t)-\rho_{1} G_{1}^{\prime}(t)
$$

$\left[\frac{K}{\pi} \frac{V_{2}^{2}}{h_{1}^{2}} k \operatorname{coth} k h_{1}-k^{2} T-g\left(\rho_{2}-\rho_{1}\right)\right] a(t)-k^{-1}$

$$
\cdot\left(\rho_{2} \operatorname{coth} k h_{2}+\rho_{1} \operatorname{coth} k h_{1}\right) \frac{d^{2} a(t)}{d t^{2}}=0,
$$

or

$$
\frac{d^{2} a}{d t^{2}}+\left(\omega_{0}^{2}-\beta-\beta \cos 2 \omega t\right) a=0
$$

in which

$$
\begin{aligned}
\omega_{0}^{2} & =\frac{k^{3} T-g k\left(\rho_{2}-\rho_{1}\right)}{\rho_{2} \operatorname{coth} k h_{2}+\rho_{1} \operatorname{coth} k h_{1}}, \\
\beta & =\frac{K V_{0}^{2} k^{2} \operatorname{coth} k h_{1}}{2 \pi h_{1}^{2}\left(\rho_{2} \operatorname{coth} k h_{2}+\rho_{1} \operatorname{coth} k h_{1}\right)} .
\end{aligned}
$$

Equation (21) can be put in the canonical form of the Mathieu equation

$$
\frac{d^{2} a}{d \tau^{2}}+(p-2 q \cos 2 \tau) a=0,
$$

in which $p$ and $q$ are not the pressure and the speed, if we put

$$
\tau=\omega t, \quad p=\frac{\omega_{0}^{2}-\beta}{\omega^{2}}, \quad q=\frac{\beta}{2 \omega^{2}} .
$$

The stability diagram for (24) is standard, and for completeness is shown in Fig. 2. It is seen that even if $\beta$ is very small there may be regions of instability. Since $V_{2}^{2}$ has a basic frequency of $2 \omega$, not $\omega$, the various regions correspond to double frequency and synchronism of the hydrodynamic oscillations in relation to $V_{2}$, instead of synchronism and half-

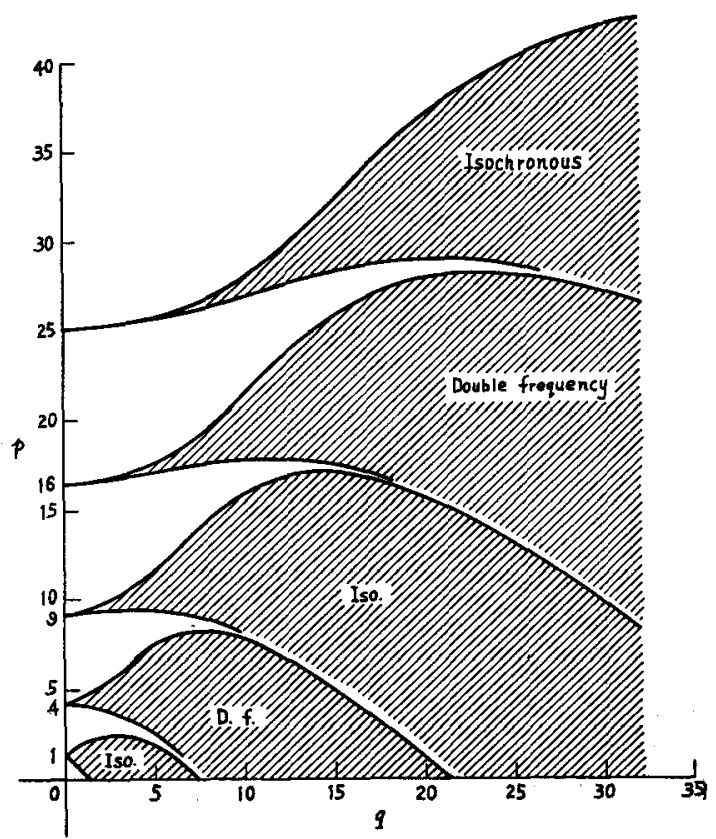

Fig. 2. The stability diagram for Mathieu's equation.

frequency, respectively. In conclusion we note that if $V_{1}$ is zero instead of $-V_{2}$, the $V_{0}$ in (23) should be replaced by $V_{0} / 2$. The instability found here is akin to the one found by Benjamin and Ursell ${ }^{2}$ for the free surface of a liquid in vertical periodic motion.

\section{ACKNOWLEDGMENTS}

This paper is a direct result of the stimulation of a public lecture given by Sir Geoffrey Taylor on October 4, 1967, which was an event in the Sesquicentennial Celebrations of The University of Michigan.

The work has been jointly sponsored by the National Science Foundation and the Army Research Office (Durham).

2 T. B. Benjamin and F. Ursell, Proc. Roy. Soc. (London) A225, 505 (1954). 